\title{
Combinatorial configurations, quasiline arrangements, and systems of curves on surfaces
}

\author{
Jürgen Bokowski \\ Department of Mathematics, Technische Universität Darmstadt, Darmstadt, Germany \\ Jurij Kovič , Tomaž Pisanski \\ University of Primorska, FAMNIT, Koper, and IMFM, Ljubljana, Slovenia \\ Arjana Žitnik \\ University of Ljubljana, FMF, and IMFM, Ljubljana, Slovenia
}

Received 18 May 2016, accepted 28 December 2016, published online 19 June 2017

\begin{abstract}
It is well known that not every combinatorial configuration admits a geometric realization with points and lines. Moreover, some of them do not even admit realizations with pseudoline arrangements, i.e., they are not topological. In this paper we generalize the concept of topological configurations to a more general one (in a least possible way) such that every combinatorial configuration is realizable in this way.

In particular, we generalize the notion of a pseudoline arrangement to the notion of a quasiline arrangement by relaxing the condition that two pseudolines meet exactly once. We also generalize well-known tools from pseudoline arrangements such as sweeps and wiring diagrams. We introduce monotone quasiline arrangements as a subfamily of quasiline arrangements that can be represented with generalized wiring diagrams. We show that every incidence structure (and therefore also every combinatorial configuration) can be realized as a monotone quasiline arrangement in the real projective plane.

A quasiline arrangement with selected vertices belonging to an incidence structure can be viewed as a map on a closed surface. Such a map can be used to distinguish between two "distinct" realizations of an incidence structure as a quasiline arrangement.

Keywords: Pseudoline arrangement, quasiline arrangement, projective plane, incidence structure, combinatorial configuration, topological configuration, geometric configuration, sweep, wiring diagram, allowable sequence of permutations, maps on surfaces.
\end{abstract}

Math. Subj. Class.: 51E20, 52C30, 05B30

E-mail addresses: juergen.bokowski@gmail.com ( Jürgen Bokowski), jurij.kovic@siol.net (Jurij Kovič), tomaz.pisanski@upr.si (Tomaž Pisanski), arjana.zitnik@fmf.uni-lj.si (Arjana Žitnik) 


\section{Introduction}

A $\left(n_{k}\right)$ configuration consists of a set of $n$ points and a set of $n$ lines, such that each point is incident to the same number $k$ of lines and each line is incident to the same number $k$ of points. We adopt the view from Grünbaum's book [12] that configurations of points and lines come in three sorts: combinatorial, geometric and topological.

The most general are combinatorial configurations where sets of points and lines are abstract sets. A configuration is geometric if its lines are lines in Euclidean or projective plane, and its points form a subset of intersection points of these lines. Similarly, a configuration is topological if its lines are pseudolines in the projective plane that form a pseudoline arrangement (formal definitions will be given in later sections). An important problem is to consider whether a given combinatorial configuration can be realized as a geometric or as a topological configuration.

The smallest combinatorial $\left(n_{3}\right)$ configuration, the Fano plane, has seven points and seven lines and is unique, up to isomorphism. Likewise, there is only one $\left(8_{3}\right)$ configuration, called Möbius-Kantor configuration. There are three nonisomorphic $\left(9_{3}\right)$ configurations, one of them is the well known Pappus configuration. While Fano plane and Möbius-Kantor configuration cannot be realized as geometric configurations (see, for example, [12, Theorem 2.1.3]), all the $\left(9_{3}\right)$ configurations can. The Pappus configuration as a geometric configuration is shown in Figure 1.

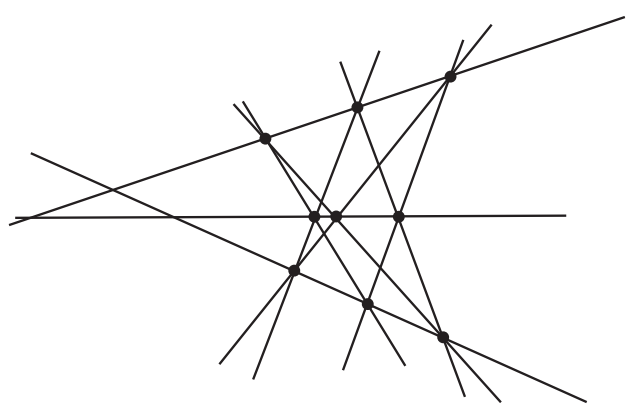

Figure 1: The Pappus configuration.

It was realized quite early by $\mathrm{H}$. Schröter [17] that among the ten $\left(10_{3}\right)$ configurations, exactly one, called anti-Desargues configuration, is not geometric. A remarkable result was proved by E. Steinitz in his thesis [18] that every combinatorial $\left(n_{3}\right)$ configuration can be represented with straight lines, except for one that may be curved. He didn't notice however, that additional incidences may appear.

F. Levi [13] introduced the notion of pseudolines and showed that the combinatorial configurations $\left(7_{3}\right)$ and $\left(8_{3}\right)$ cannot be realized with pseudoline arrangements in the projective plane. There is a fundamental difference between the $\left(7_{3}\right)$ and $\left(8_{3}\right)$ configurations and the anti-Desargues configuration. Namely, the latter can be realized as a pseudoline arrangement in the projective plane, i.e. it is a topological configuration. J. Bokowski and his coworkers applied modern methods of computational synthetic geometry to study the existence or nonexistence of topological configurations [5]. B. Grünbaum, J. Bokowski, and L. Schewe [1] investigated the problem of existence of topological $\left(n_{4}\right)$ configurations and showed that topological configurations exist if and only if $n \geq 17$. Recently J. Bokowski 
and R. Strausz [4] associated to each topological configuration a map on a surface that they call a manifold associated to the topological configuration and suggested a possible definition of equivalence of two topological configurations. Namely, topological configurations are distinct in a well-defined sense if and only if the associated maps are distinct.

The main purpose of this paper is to generalize the concept of topological configurations to a more general one (in the least possible way) such that every combinatorial configuration, and more generally, every combinatorial incidence structure, would be realizable in this sense. It is not hard to see that every combinatorial incidence structure can be realized by points and curves in the projective plane. However, it is not obvious that all the curves in question may, in fact, be pseudolines. It turns out that we only need to relax the condition of unique intersection of pairs of pseudolines.

In Section 4 we first generalize the notion of a pseudoline arrangement to the notion of a quasiline arrangement by relaxing the condition that two pseudolines meet exactly once. Then we introduce a subclass of quasiline arrangements that we call monotone. In Sections 5 and 6 we also generalize well-known tools from pseudoline arrangements such as sweeps, wiring diagrams, and allowable sequences of permutations. It is known that every pseudoline arrangement can be represented by a wiring diagram and conversely, every wiring diagram can be viewed as a pseudoline arrangement; see J. E. Goodman [8]. We show that every monotone quasiline arrangement can be represented by a generalized wiring diagram that is in turn also a monotone quasiline arrangement. In this respect the class of monotone quasiline arrangements is the weakest generalization of the class of pseudoline arrangements.

In Section 7 we deal with polygonal quasiline arrangements. We show that a monotone quasiline arrangement without digons is topologically equivalent to a monotone polygonal quasiline arrangement with no bends (arcs connecting two vertices of the arrangement are all straight lines).

In Section 8 we introduce a generalization of topological incidence structures that we call (monotone) quasi-topological incidence structures by allowing the set of lines to form a (monotone) quasiline arrangement instead of a pseudoline arrangement. In Section 9 we show the main result of this paper that every combinatorial incidence structure, in particular every combinatorial configuration, can be realized as a monotone quasi-topological incidence structure.

A natural parameter for a monotone quasiline arrangement is the maximal number of crossings of pairs of pseudolines. A monotone quasiline arrangement is a pseudoline arrangement if and only if this parameter is equal to 1 . Similarly, combinatorial incidence structures can be stratified by the minimal number of crossings over all the representations as monotone quasi-topological incidence structures. Topological incidence structures are exactly those representations with the maximal number of crossings equal to 1 . The proof of our main result is constructive and it shows that an incidence structure with $v$ points and $n$ lines can be realized such that the number of intersections of every two pseudolines is upper bounded by $(v+1) n^{2}$. It would be interesting to characterize those incidence structures which allow realizations with monotone quasiline arrangements such that the maximal number of intersections of two pseudolines is small and/or independent of $v$ and $n$.

Finally, the concept of a map associated with a topological configuration can be extended to the quasi-topological case (Section 10). 


\section{Combinatorial and geometric incidence structures}

In this section we review basic definitions and facts about incidence structures; see B. Grünbaum [12] or T. Pisanski and B. Servatius [15] for more background information. An incidence structure $\mathcal{C}$ is a triple $\mathcal{C}=(\mathcal{P}, \mathcal{L}, \mathcal{I})$, where $\mathcal{P}$ and $\mathcal{L}$ are non-empty disjoint finite sets and $\mathcal{I} \subseteq \mathcal{P} \times \mathcal{L}$. The elements of $\mathcal{P}$ are called points and the elements of $\mathcal{L}$ are called lines. The relation $\mathcal{I}$ is called incidence relation; if $(p, L) \in \mathcal{I}$, we say that the point $p$ is incident to the line $L$ or, in a geometrical language, that $p$ lies on $L$. We further require that each point lies on at least two lines and each line contains at least two points. To stress the fact that these objects are of purely combinatorial nature we sometimes call them abstract or combinatorial incidence structures.

Two incidence structures $\mathcal{C}=(\mathcal{P}, \mathcal{L}, \mathcal{I})$ and $\mathcal{C}^{\prime}=\left(\mathcal{P}^{\prime}, \mathcal{L}^{\prime}, \mathcal{I}^{\prime}\right)$ are isomorphic, if there exists an incidence preserving bijective mapping from $\mathcal{P} \cup \mathcal{L}$ to $\mathcal{P}^{\prime} \cup \mathcal{L}^{\prime}$ which maps $\mathcal{P}$ to $\mathcal{P}^{\prime}$ and $\mathcal{L}$ to $\mathcal{L}^{\prime}$.

Complete information about the incidence structure can be recovered also from its Levi graph with a given black and white coloring of the vertices. The Levi graph $G(\mathcal{C})$ of an incidence structure $\mathcal{C}$ is a bipartite graph with "black" vertices $\mathcal{P}$ and "white" vertices $\mathcal{L}$ and with an edge joining some $p \in \mathcal{P}$ and some $L \in \mathcal{L}$ if and only if $p$ lies on $L$ in $\mathcal{C}$.

An incidence structure is lineal if any two distinct points are incident with at most one common line (or equivalently, any two distinct lines are incident to at most one point). This is equivalent to saying that the Levi graph of a lineal incidence structure has girth at least 6. A $\left(n_{r}, b_{k}\right)$-configuration is a lineal incidence structure $\mathcal{C}=(\mathcal{P}, \mathcal{L}, \mathcal{I})$ with $|\mathcal{P}|=v$ and $|\mathcal{L}|=b$ such that each line is incident with the same number $k$ of points and each point is incident with the same number $r$ of lines. In the special case when $n=b$ (and by a simple counting argument also $r=k$ ) we speak of a balanced configuration and shorten the notation $\left(n_{k}, n_{k}\right)$ to $\left(n_{k}\right)$.

A set of lines in the real Euclidean or projective plane together with a subset of intersection points of these lines such that each line contains at least two intersection points is called a geometric incidence structure. From the definition it follows that each point lies on at least two lines. A geometric incidence structure together with the incidences of points and lines defines a combinatorial incidence structure, which we call the underlying combinatorial incidence structure. Such a combinatorial incidence structure is certainly lineal. Two geometric incidence structures are isomorphic if their underlying combinatorial incidence structures are isomorphic. A geometric incidence structure $\mathcal{G}$ is a realization of a combinatorial incidence structure $\mathcal{C}$ if the underlying combinatorial incidence structure of $\mathcal{G}$ is isomorphic to $\mathcal{C}$.

\section{Pseudolines and topological incidence structures}

In this section we review basic facts about pseudoline arrangements. For more background on this topic see J. E. Goodman [8] or B. Grünbaum [11]. By a projective plane we mean the real projective plane or the extended Euclidean plane. A pseudoline is a simple noncontractible closed curve in the projective plane. In particular, each line in the projective plane is a pseudoline.

Pseudolines and certain relationships between them inherit properties from the topological structure of the projective plane [8]. For instance:

Fact I. Any two pseudolines have at least one point in common. 
Fact II. If two pseudolines meet in exactly one point they intersect transversally at that point.

A pseudoline arrangement $\mathcal{A}$ is a collection of at least two pseudolines in the projective plane with the property that each pair of pseudolines of $\mathcal{A}$ has exactly one point in common (at which they cross transversally). Such a point of intersection is called a vertex or a crossing of the arrangement. A crossing in which only two pseudolines meet is called regular. If more than two pseudolines meet in the same point, the crossing is called singular. Each pseudoline arrangement $\mathcal{A}$ determines an associated 2-dimensional cell complex into which the pseudolines of $\mathcal{A}$ decompose the projective plane. Its cells of dimension $0,1,2$ are called vertices, edges, and cells (or polygons), respectively; see [11, p. 40]. Two pseudoline arrangements are isomorphic if the associated cell complexes are isomorphic; that is, if and only if there exists an incidence preserving bijective mapping between the vertices, edges, and cells of one arrangement and those of the other.

We say that a pseudoline is polygonal, if it is a line or it can be subdivided into a finite number of closed line segments (whereby the endpoints of the line segments occur of course twice). A pseudoline arrangement is polygonal if every pseudoline of the arrangement is polygonal. Note that a line arrangement is polygonal. A point on a polygonal pseudoline that is not a crossing of the arrangement is called a bend if two line segments meet at that point and the join of these segments is not again a line segment. A crossing $v$ of a polygonal pseudoline arrangement is straight, if there exists a neighborhood $N(v)$ of $v$ such that the intersection of $N(v)$ with every pseudoline $\ell$ containing $v$ is a line segment.

It is well-known that every pseudoline arrangement is isomorphic to a a polygonal pseudoline arangement. In [11, Theorem 3.3] essentialy the following theorem is presented and a proof by induction is proposed.

Proposition 3.1. Every pseudoline arrangement is isomorphic to a polygonal pseudoline arrangement with no bends.

To describe pseudoline arrangements combinatorially, wiring diagrams are standard tools to use; see J. E. Goodman [8] and J. E. Goodman and R. Pollack [9].

A partial wiring diagram is a collection of $x$-monotone polygonal lines, called wires, in the Euclidean plane, each of them horizontal except for a finite number of "short" segments, where it crosses another polygonal line. A wiring diagram is a partial wiring diagram with the property that every two polygonal lines cross exactly once.

A wiring diagram whose wires are equally spaced can be viewed as a pseudoline arrangement: take a disk that is large enough that all the crossings are in its interior, and positioned such that the intersections of each polygonal line with the boundary of the disk are on opposite sides of the boundary of the disk. The disk can now be viewed as a disk model of the projective plane and the lines of the wiring diagram inside the disk form a polygonal pseudoline arrangement. Figure 2 (b) shows a wiring diagram that is isomorphic to the line arrangement on Figure 2 (a).

Conversely, every pseudoline arrangement can be described with a wiring diagram; see [8].

\section{Theorem 3.2. Every pseudoline arrangement is isomorphic to a wiring diagram.}

A pseudoline arrangement can also be viewed as an incidence structure when we define a subset of its crossings as its point set. We say that an incidence structure is topological if 

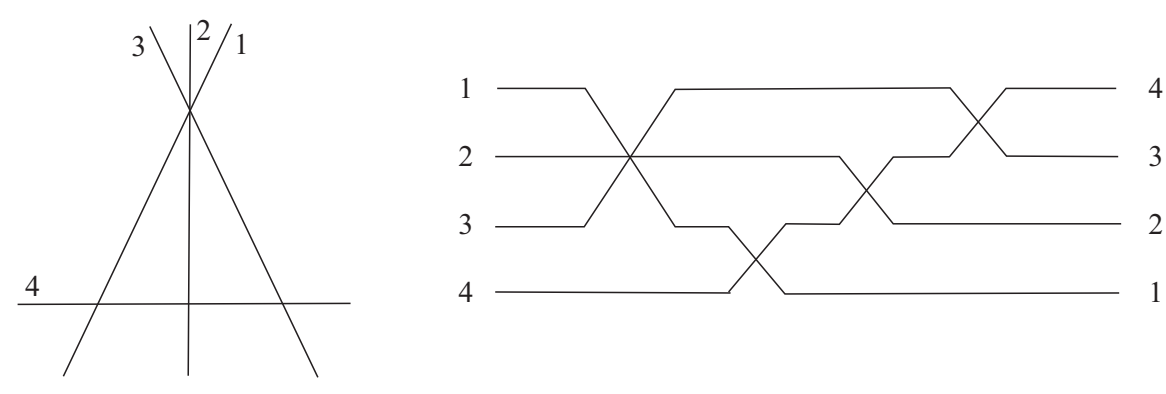

Figure 2: A pseudoline arrangement and a corresponding wiring diagram.

its points are points in the projective plane, and lines are pseudolines that form a pseudoline arrangement. A topological incidence structure $\mathcal{T}$ is a topological realization of a combinatorial incidence structure $\mathcal{C}$ if the underlying combinatorial incidence structure of $\mathcal{T}$ is isomorphic to $\mathcal{C}$. A topological incidence structure is polygonal if its lines are polygonal pseudolines. Note that any geometric incidence structure is also (polygonal) topological.

There are three distinct notions of equivalence of topological incidence structures. The weakest is combinatorial equivalence. Two topological incidence structures are combinatorially equivalent or isomorphic if they are isomorphic as combinatorial incidence structures. The strongest one is the notion of topological equivalence between pseudoline arrangements in the projective plane. Two topological incidence structures are topologically equivalent if there exists an isomorphism of the underlying pseudoline arrangements that induces an isomorphism of the underlying combinatorial incidence structures.

One intermediate notion is mutation equivalence; see J. Bokowski and V. Pilaud [2]. A mutation or a Reidemeister move of a pseudoline arrangement is a local transformation to another pseudoline arrangement where only one pseudoline $\ell$ moves across a single crossing $v$ of the remaining arrangement. Only the position of the crossings of $\ell$ with the pseudolines incident to $v$ is changed. If those crossings are not points of the incidence structure, we say that such a mutation is admissible. Two topological incidence structures are mutation equivalent if they can be modified by (possibly empty) sequences of admissible mutations to obtain topologically equivalent topological incidence structures.

As a consequence of Proposition 3.1 we have the following result.

Proposition 3.3. Every topological incidence structure is topologically equivalent to a polygonal topological incidence structure with no bends.

As we mentioned already, the underlying combinatorial incidence structure of a geometric incidence structure is necessary lineal. Note that the same is true for the underlying combinatorial incidence structure of a topological incidence structure. This is equivalent to saying that every incidence structure that allows a description with a wiring diagram is lineal. However, our generalization considered in the next sections covers both lineal and non-lineal incidence structures. 


\section{Quasiline arrangements}

In this section we generalize the notion of a pseudoline arrangement in which we relax the condition on pseudoline crossings. A quasiline arrangement is a collection of at least two pseudolines in the real projective plane with the property that any two pseudolines have a finite number of points in common and that at each common point they cross transversally. Note that any pair of pseudolines in a quasiline arrangement meets an odd number of times. The terms such as crossings, bends, polygonal quasiline arrangements, and isomorphic quasiline arrangements are defined in the same way as for pseudoline arrangements.

To simplify the discussion we will consider only quasiline arrangements in the extended Euclidean plane with the following additional properties:

- none of the crossings of the arrangement are points at infinity and

- every pseudoline of the arrangement intersects the line at infinity exactly once.

The former condition can easily be achieved in general by a suitable projective transformation while the latter is an essential assumption. Namely, a pseudoline can intersect the line at infinity more than once.

In the sequel we define a subclass of quasiline arrangements, called monotone quasiline arrangements, that is in some sense the least generalization of the pseudoline arrangements.

It is well-known that the extended Euclidean plane is in its topological sense equivalent to the disk model of the projective plane: the projective plane is represented by a disk, with all the pairs of antipodal points on the boundary identified. The boundary of the disk is the line at infinity of the projective plane and the points on the line at infinity are points at infinity. For the rest of the section we will use the disk model of the projective plane.

Let $\mathcal{A}$ be a quasiline arrangement. We choose an orientation for the line at infinity $\ell$ and a point $x$ on $\ell$ that is not on any of the pseudolines of the arrangement. Denote by $x^{+}$, $x^{-}$the corresponding points on the boundary of the disk representing the projective plane without identifying $x^{+}$and $x^{-}$. These two points divide the boundary of the disk into two arcs, $\ell^{+}$from $x^{+}$to $x^{-}$and $\ell^{-}$from $x^{-}$to $x^{+}$, again by forgetting the identification of antipodal points. Starting at $x^{+}$and moving along $\ell$ in the positive direction we orient a pseudoline that we meet for the first time such that it points to the interior of the circle. We call such an orientation a monotone orientation of the quasiline arrangement $\mathcal{A}$ and call the arrangement together with the point $x^{+}$(or equivalently, with a monotone orientation) a marked arrangement. In a marked arrangement we have a natural ordering of the pseudolines as the order of intersections with $\ell$ starting at $x^{+}$. Moreover, the orientation of pseudolines induces the order of crossings on every pseudoline. A marked arrangement $\left(\mathcal{A}, x^{+}\right)$is proper if the order of the intersection points of any two pseudolines is the same for both pseudolines. Observe that the property of being proper is heredity by taking subarrangements.

A quasiline arrangement $\mathcal{A}$ is a monotone quasiline arrangement if there exists a proper marked arrangement $\left(\mathcal{A}, x^{+}\right)$for some $x^{+}$on the boundary of the disk, representing the line at infinity. Note that it is not the same to require that there exists an orientation of the pseudolines such that the order of the intersection points on any two pseudolines is the same for both pseudolines. Figure 3 shows a quasiline arrangement in which the order of crossings is the same for any pair of pseudolines if the pseudolines are oriented alternatingly. However, it is not a monotone quasiline arrangement, since any monotone orientation of the arrangement induces different orders of crossings on the horizontal line and at least one of the other pseudolines. 


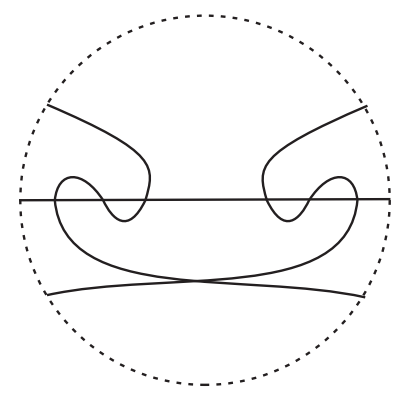

Figure 3: A quasiline arrangement that is not a monotone quasiline arrangement.

We now generalize the notion of the wiring diagram from Section 3 to be able to describe also monotone quasiline arrangements. A generalized wiring diagram is a partial wiring diagram with the property that every two polygonal lines cross an odd number of times. A generalized wiring diagram can be viewed as a monotone polygonal quasiline arrangement just like a wiring diagram can be viewed as a pseudoline arrangement. Figure 4 (b) shows a generalized wiring diagram which is topologically equivalent to the quasiline arrangement from Figure 4 (a). Observe that by adding seven points (corresponding to the crossings where three pseudolines cross) we arrive at the unique $\left(7_{3}\right)$ configuration the Fano plane.

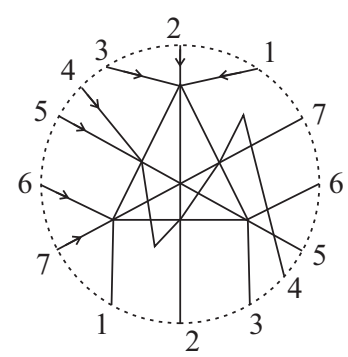

(a)

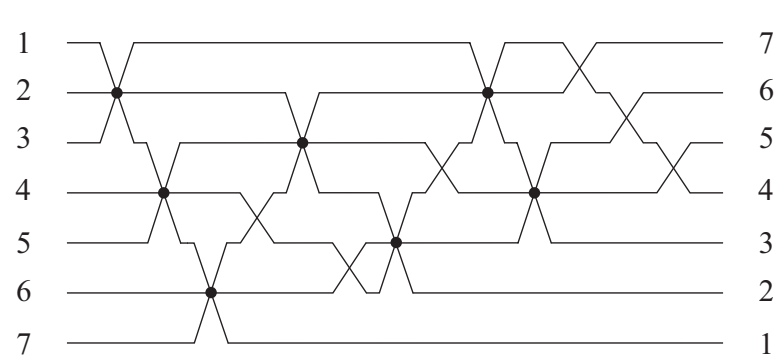

(b)

Figure 4: (a) A monotone quasiline arrangement and (b) its generalized wiring diagram.

One of the main results of the paper is the following generalization of Theorem 3.2.

Theorem 4.1. Every monotone quasiline arrangement is isomorphic to a generalized wiring diagram.

We will prove the theorem in Section 6. To do this we will need the notions of sweeping and allowable sequences of permutations, which we introduce in the next two sections.

\section{Sweeping quasiline arrangements}

In this section we introduce the notion of sweeping and show that every proper marked quasiline arrangement has a sweep. With a slight modification working in the disk model of the projective plane instead of the Euclidean plane, we follow S. Felsner and H. Weil [7]. 
Let $\left(\mathcal{A}, x^{+}\right)$be a proper marked quasiline arrangement. A sweep of $\left(\mathcal{A}, x^{+}\right)$is a sequence $c_{0}, c_{1}, \ldots, c_{r}$, of pseudolines such that the following conditions hold:

(1) pseudolines $c_{0}$ and $c_{r}$ coincide with the line at infinity,

(2) any two pseudolines $c_{i}$ and $c_{j}$ intersect exactly once at $x$,

(3) none of the pseudolines $c_{i}$ contains a vertex of the arrangement $\mathcal{A}$,

(4) each pseudoline $c_{i}$ has exactly one point of intersection with each pseudoline of $\mathcal{A}$,

(5) for any two consecutive pseudolines $c_{i}, c_{i+1}$ of the sequence there is exactly one vertex of the arrangement $\mathcal{A}$ between them, i.e., in the interior of the region bounded by $c_{i}$ and $c_{i+1}$ disjoint from the line at infinity.

We will show that every proper marked quasiline arrangment has a sweep. To this end we define a directed graph, or briefly a digraph, $D=D\left(\mathcal{A}, x^{+}\right)$that corresponds to a marked quasiline arrangement $\left(\mathcal{A}, x^{+}\right)$as follows. The vertices of $D$ are the vertices of $\mathcal{A}$ and there is a directed edge for every pair of vertices $u$ and $v$ that are consecutive on an arc of some pseudoline oriented from $u$ to $v$ that has an empty intersection with the line at infinity. We say that such a digraph is associated with the marked arrangement $\left(\mathcal{A}, x^{+}\right)$. Note that this digraph is embedded in the plane. The undirected plane graph $G$ underlying $D\left(\mathcal{A}, x^{+}\right)$will be called the graph associated with the arrangement $\mathcal{A}$. Note that $G$ depends only on the arrangement $\mathcal{A}$ and not on the orientation of the pseudolines.

In [7] S. Felsner and H. Weil prove that the digraph associated with a marked arrangement of pseudolines is acyclic. We prove the following generalization of this result.

Lemma 5.1. The digraph associated with a proper marked quasiline arrangement is acyclic.

Proof. Without loss of generality we may assume that the line at infinity is oriented counterclockwise. Let $\left(\mathcal{A}, x^{+}\right)$be a proper marked quasiline arrangement and $D$ its associated digraph. Note that $D$ is a plane graph, embedded in the interior of the disk, representing the projective plane. The interior of the disk is homeomorphic to the plane, therefore Jordan curve theorem applies; see [14, p. 25].

First we observe that $D$ contains no directed cycles of length 2 , otherwise there are two lines with different orders of vertices on them and $\left(\mathcal{A}, x^{+}\right)$is not a proper marked arrangement. For the same reason there are no directed cycles, all the edges of which belong to two pseudolines.

Suppose $D$ contains a directed cycle. Let $C$ be a directed cycle, given by the sequence of vertices and edges $v_{0}, e_{0}, v_{1}, \ldots, e_{t-1}, v_{t}=v_{0}$ such that no other directed cycle is contained in the area bounded by $C$. It is easy to see that $C$ bounds a face of $D$ by Jordan's theorem. Since at each vertex of the arrangement there meet at least two pseudolines, two consecutive edges of $C$ lie on different pseudolines. Assume that $C$ is oriented clockwise; see Figure 5. If $C$ is oriented counterclockwise, the proof is similar.

Now consider the arrangement $\mathcal{A}^{\prime}$ consisting only of the pseudolines $\ell_{0}, \ldots, \ell_{t-1}$ that contain the edges $e_{0}, \ldots, e_{t-1}$ of $C$ consecutively. Note that also $\left(\mathcal{A}^{\prime}, x^{+}\right)$is a proper marked arrangement. Denote by $p_{i}$ the intersection of $\ell_{i}$ with the line at infinity $\ell$ for each $i$. We observe that $\ell_{i}$ and $\ell_{i+1}$ are distinct (the indices are taken modulo $t$ ), since two consecutive edges of $C$ lie on different pseudolines. Without loss of generality we may assume that $p_{0}^{+}$appears first after $x^{+}$. 
Since $p_{1}^{+}$appears after $p_{0}^{+}$on the line at infinity, the arc of $\ell_{1}$ from $v_{1}$ to $p_{1}^{+}$must intersect $\ell_{0}$ to reach $\ell^{+}$. It can intersect $\ell_{0}$ only between $p_{0}^{+}$and $v_{0}$ (an odd number of times), otherwise we obtain a directed cycle contained in only two pseudolines. Similarly, since $p_{1}^{-}$appears after $p_{0}^{-}$on $\ell^{-}$, the arc of $\ell_{1}$ from $v_{2}$ to $p_{1}^{-}$must intersect $\ell_{0}$ to reach $\ell^{-}$. It can intersect $\ell_{0}$ only between $v_{1}$ and $p_{0}^{-}$(an odd number of times); see Figure 5.

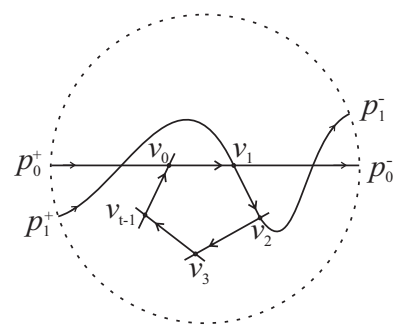

Figure 5: A directed cycle in a marked quasiline arrangement.

Now the arc of $\ell_{2}$ between $v_{2}$ and $p_{2}^{+}$cannot intersect $\ell_{1}$ after $v_{2}$, since then we again obtain a directed cycle on two lines. Therefore it must intersect $\ell_{0}$ after $v_{1}$ and then again before $v_{0}$ to reach $\ell^{+}$. If $t>2$, we see that also the arc of $\ell_{2}$ between $v_{3}$ and $p_{2}^{-}$must intersect $\ell_{0}$ to reach $\ell^{-}$. In order to avoid self-intersection it can cross $\ell_{0}$ only after $v_{1}$ (at least once). With the same reasoning we conclude that for each of $\ell_{i}, i=2, \ldots, t-2$, either $\ell_{i}=\ell_{0}$ or

- the arc of $\ell_{i}$ between $v_{i}$ and $p_{i}^{+}$first intersects $\ell_{0}$ after $v_{1}$ at least once and then again before $v_{0}$ at least once;

- the arc of $\ell_{i}$ between $v_{i+1}$ and $p_{i}^{-}$intersects $\ell_{0}$ only after $v_{1}$ (at least once).

For the line $\ell_{t-1}$ there are two cases to consider.

- The pseudolines $\ell_{t-1}$ and $\ell_{1}$ are distinct. As before, the arc of $\ell_{t-1}$ between $v_{t-1}$ and $p_{t-1}^{+}$first intersects $\ell_{0}$ after $v_{1}$ at least once and then again before $v_{0}$ at least once. But then the arc of $\ell_{t-1}$ after $v_{0}$ cannot reach $\ell^{-}$without intersecting $\ell_{0}$ before $v_{0}$ thus producing a directed cycle on two pseudolines, or self-intersection. A contradiction.

- The pseudoline $\ell_{t-1}$ is the same as $\ell_{1}$. Then the order of vertices $v_{0}=v_{t}$ and $v_{1}$ is not the same for $\ell_{0}$ and $\ell_{1}$, a contradiction.

Lemma 5.2. Every proper marked quasiline arrangement has a sweep.

Proof. Let $\left(\mathcal{A}, x^{+}\right)$be a proper marked quasiline arrangement. By Lemma 5.1, its associated digraph $D$ is acyclic. Therefore there exist a topological sorting $v_{1}, \ldots, v_{r}$ of the vertices of $D$.

We will define a sweep consisting of pseudolines $c_{0}, c_{1}, \ldots, c_{r}$ passing through $x$, such that in the interior of the region bounded by $c_{i-1}$ and $c_{i}, i=1, \ldots, r$, there will be exactly one vertex of the arrangement, namely $v_{i}$.

Define $c_{0}$ and $c_{r}$ to be the line at infinity.

Suppose that $c_{i-1}$ has been defined for some $i<r-1$. Let $\ell_{i_{1}}, \ldots, \ell_{i_{t}}$ be the pseudolines of the arrangement $\mathcal{A}$ that contain $v_{i}$, in the order they intersect $c_{i-1}$. Take the triangle 
$T$ with sides on $c_{i-1}, \ell_{i_{1}}$ and $\ell_{i_{t}}$ and one of the vertices being $v_{i}$. This is well defined, since $\ell_{i_{1}}$ and $\ell_{i_{t}}$ intersect $c_{i-1}$ only once by definition of $c_{i-1}$. Only vertices $v_{1}, \ldots, v_{i-1}$ of the arrangement (and all of them) are on the other side of $c_{i-1}$ as $v_{i}$ (in the interior of the disk, representing the projective plane), therefore all the lines $\ell_{i_{1}}, \ldots, \ell_{i_{1}}$ are directed towards $v_{i}$ and there are no other vertices of the arrangement in the triangle $T$ besides $v_{i}$. Define $c_{i}$ to be the right boundary of an $\epsilon$-tube around $c_{i-1}$ and $T$, until it approaches $x$. If $\epsilon$ is small enough, only vertex $v_{i}$ will be in the interior of the region bounded by $c_{i-1}$ and $c_{i}$ and $c_{i}$ will intersect every line of the arrangement only once.

Clearly the pseudolines $c_{0}, c_{1}, \ldots, c_{r}$ obtained in this way define a sweep of the marked arrangement $\left(\mathcal{A}, x^{+}\right)$.

\section{Generalized allowable sequences and wiring diagrams}

Wiring diagrams and allowable sequences of permutations are standard tools for describing pseudoline arrangements; see J. E. Goodman [8] and J. E. Goodman and R. Pollack [9]. We need to generalize these two notions in order to be able to describe also monotone quasiline arrangements. We introduced generalized wiring diagrams in Section 4. In this section we generalize also the notion of an allowable sequence and show how generalized wiring diagrams and generalized allowable sequences of permutations are related.

Fix $n \in \mathbb{N}$. A sequence $\Sigma=\pi_{0}, \ldots, \pi_{r}$ of permutations is called a partial allowable sequence of permutations if it fulfills the following properties:

(1) $\pi_{0}$ is the identity permutation on $\{1, \ldots, n\}$,

(2) each permutation $\pi_{i}, 1 \leq i \leq r$, is obtained by the reversal of a consecutive substring $M_{i}$ from the preceding permutation $\pi_{i-1}$.

For every $i, 1 \leq i \leq r$, we call $M_{i}$ a move. Move $M_{i}$ represents the transition from permutation $\pi_{i-1}$ to permutation $\pi_{i}$.

Two partial allowable sequences $\Sigma$ and $\Sigma^{\prime}$ are elementary equivalent if $\Sigma$ can be transformed into $\Sigma^{\prime}$ by interchanging two disjoint adjacent moves. Two partial allowable sequences $\Sigma$ and $\Sigma^{\prime}$ are called equivalent if there exists a sequence $\Sigma=\Sigma_{1}, \Sigma_{2}, \ldots, \Sigma_{m}=$ $\Sigma^{\prime}$ of partial allowable sequences such that $\Sigma_{i}$ and $\Sigma_{i+1}$ are elementary equivalent for $1 \leq i<m$.

A partial allowable sequence of permutations of $n$ elements is called an allowable sequence of permutations if any two elements $x, y \in\{1, \ldots n\}$ are joint members of exactly one move. The following result is easy to see.

Proposition 6.1. Let $\Sigma=\pi_{0}, \ldots, \pi_{r}$ be an allowable sequence of permutations. Then $\pi_{r}$ is the reverse permutation on $\{1, \ldots, n\}$.

A partial allowable sequence of permutations $\Sigma=\pi_{0}, \ldots, \pi_{r}$ is called a qeneralized allowable sequence of permutations if any two elements $x, y \in\{1, \ldots n\}$ are joint members of an odd number of moves. We make the following observation.

Proposition 6.2. A partial allowable sequence of permutations $\Sigma=\pi_{0}, \ldots, \pi_{r}$ is a generalized allowable sequence of permutations if and only if $\pi_{r}$ is the reverse permutation on $\{1, \ldots, n\}$.

To any partial allowable sequence there corresponds a partial wiring diagram: 
- start drawing $n$ horizontal lines, numbered with numbers $1, \ldots, n$ from top to bottom, at points $(0, n), \ldots,(0,1)$,

- to each move $M_{i}$ there corresponds coordinate $x_{i}=i$,

- at each coordinate $x_{i}$ there is a crossing where the lines in the move $M_{i}$ cross transversally; i.e., the order of lines from $M_{i}$ is reversed.

Conversely, to any partial wiring diagram there corresponds a partial allowable sequence in the following way. We number the lines of the wiring diagram from top to bottom with numbers $1, \ldots, n$. We start with the identity permutation and after each crossing of the wiring diagram we list the lines from top to bottom.

Obviously, a partial wiring diagram, corresponding to an allowable sequence is also a wiring diagram. A partial wiring diagram, corresponding to a generalized allowable sequence is also a generalized wiring diagram.

Example 6.3. The sequence of permutations

$1234,3214,3241,3421,4321$

is an allowable sequence of permutations which corresponds to the wiring diagram from Figure 2.

Now we can prove Theorem 4.1.

Proof of Theorem 4.1. Let $\mathcal{A}$ be a monotone quasiline arrangement with $n$ pseudolines. Then there exists a proper marked arrangement $\left(\mathcal{A}, x^{+}\right)$for some $x$ on the line at infinity. We may label the lines of $\mathcal{A}$ by numbers $1, \ldots, n$ in the order in which they are met on the line at infinity starting at $x^{+}$. Take a sweep $c_{0}, c_{1}, \ldots, c_{r}$ of $\left(\mathcal{A}, x^{+}\right)$. It determines a sequence of permutations on the set $\{1, \ldots, n\}$ : just list the lines in the order in which they are met on each $c_{i}, i=0, \ldots, r$, starting at $x^{+}$. Since between any two pseudolines $c_{i}$ and $c_{i+1}$ there is exactly one vertex of the arrangement, and the order of lines that meet in that vertex is reversed when they leave the vertex, this sequence of permutations is a partial allowable sequence of permutations. Moreover, since the order of the lines of $\mathcal{A}$ is reversed at $c_{r}$, it is also a generalized allowable sequence of permutations. To a generalized allowable sequence of permutations there corresponds a generalized wiring diagram. This generalized wiring diagram is isomorphic to the original quasiline arrangement by construction.

\section{Polygonal quasiline arrangements without bends}

In this section we return to the extended Euclidean plane, where the concept of straight lines and bends is well defined. Two distinct lines that are parallel in the Euclidean plane meet at the line at infinity. This is a bend at infinity. The notions of proper marked arrangements and monotone quasiline arrangements are also well defined in the extended Euclidean plane, by mapping homeomorphically a quasiline arrangement to the disk model of the projective plane and back, sending the line at infinity to the line at infinity.

Every pseudoline arrangement is isomorphic to a polygonal pseudoline arrangement with no bends by Proposition 3.1. With polygonal quasiline arrangements we cannot always avoid bends. The reason is that a quasiline arrangement may contain digons. A digon in a quasiline arrangement is cell of the associated cell complex that is adjacent to two 
edges and two vertices. For example, if a quasiline arrangement consists of two polygonal pseudolines, intersecting in three points, it contains three digons. We will show that digons are in fact the only reason for the need of bends in monotone polygonal quasiline arrangements. First we prove a technical lemma.

Lemma 7.1. Let $\left(\mathcal{A}, x^{+}\right)$be a proper marked quasiline arrangement that contains no digons. Let $G$ be the undirected plane graph underlying the associated directed graph $D=D\left(\mathcal{A}, x^{+}\right)$of $\left(\mathcal{A}, x^{+}\right)$. Then the following properties hold.

(i) Graph $G$ contains no cycles of length two, i.e., $G$ is simple.

(ii) Suppose $\mathcal{A}$ contains at least three crossings. Then $G$ is 2-connected.

Proof. (i) We will show that if $G$ contains a cycle of length two, then it contains a face of length two which corresponds to a digon in $\mathcal{A}$. Suppose $G$ contains a cycle $C$ of length two with vertices $v_{1}$ and $v_{2}$. If $C$ is the boundary of a face, we are done. Otherwise at least one pseudoline connects vertices $v_{1}$ and $v_{2}$ in the interior of $C$. If there are no vertices of the arrangement in the interior of $C$, we have at least two faces of length two in $G$. Otherwise there is a vertex inside $C$ where at least two pseudolines cross. To form a crossing, at least two pseudolines that are consecutive in the cyclic order around $v_{1}$ must cross. At least one crossing $w$ will be such that there is no other crossings between $v_{1}$ and $w$ on two consecutive lines through $v_{1}$. They form a face of length two of $G$ inside $C$ with vertices $v_{1}$ and $w$.

(ii) Graph $G$ is connected, since every two pseudolines of $\mathcal{A}$ cross. Let $v$ be a vertex of $G$ and suppose that $G-v$ is not connected. Then there exist vertices $u, w$ which are in different components of $G-v$. If there existed two lines of $\mathcal{A}$ not incident with $v$, one of them incident with $u$ and the other one incident with $w$ (or one line not incident with $v$, and incident with both $u$ and $w$ ) we would have a path connecting $u$ and $w$ in $G-v$. Therefore we may assume w.l.o.g. that all lines of $\mathcal{A}$ incident with $u$ are also incident with $v$ (there are at least two such lines, which intersect at least three times, once in $u$ and once in $v$ ). Since $\mathcal{A}$ contains no digons, there exists a line $\ell$ that intersects some lines through $u$ between $u$ and $v$ and is not incident with $v$ by a similar reasoning as in (i). Such a line intersects all lines through $w$. If there exists a line through $w$ not incident with $v$, we have a path from $u$ to $w$ in $G-v$, a contradiction.

If also every line incident with $w$ is incident with $v$, there exists a line $\ell^{\prime}$ that intersects some lines through $w$ between $v$ and $w$ and is not incident with $v$, otherwise we have digons. Lines $\ell$ and $\ell^{\prime}$ either intersect or $\ell$ is equal to $\ell^{\prime}$ and we again have a path from $u$ to $w$ in $G-v$, a contradiction.

Remark 7.2. Note that the requirement that the order of the intersection points of any two lines is the same for both lines in Lemma 7.1 is necessary, since otherwise there may be a vertex of the arrangement that is incident to all the lines of the arrangement and no two pseudolines that are consecutive around that vertex form a digon; see Figure 6. Note that the graph associated to this quasiline arrangement is not 2-connected.

Theorem 7.3. Let $\mathcal{A}$ be a monotone quasiline arrangement. Then $\mathcal{A}$ is isomorphic to a monotone polygonal quasiline arrangement with no bends if and only if it contains no digons. 


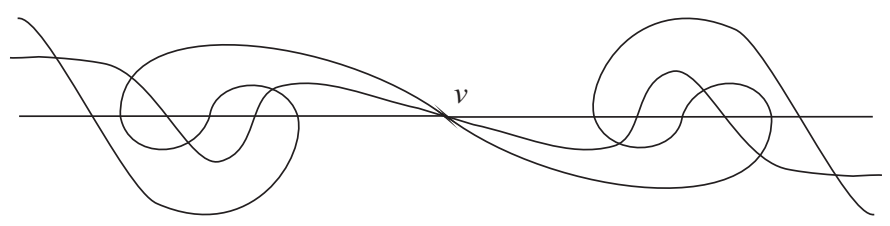

Figure 6: A quasiline arrangement such that the associated graph is not 2-connected.

Proof. If $\mathcal{A}$ contains a digon, there has to be a bend.

We now prove the converse. Suppose $\mathcal{A}$ contains no digons. If $\mathcal{A}$ contains only one crossing, the claim is clear. Therefore we may assume that $\mathcal{A}$ contains at least three crossings. Since $\mathcal{A}$ is a monotone quasiline arrangement, we may choose a point $x^{+}$such that $\left(\mathcal{A}, x^{+}\right)$is a proper marked arrangement. Let $G$ be the undirected plane graph underlying its associated directed graph $D=D\left(\mathcal{A}, x^{+}\right)$. Graph $G$ is simple and 2-connected by Lemma 7.1. Therefore we can draw $G$ in the plane in such a way that the vertices on the boundary of the outer face are the vertices of a convex polygon $P$, all the edges of $G$ are straight lines and the cyclic orders of edges around each vertex is preserved by [6]. To obtain a polygonal quasiline arrangement that is topologically equivalent to $\mathcal{A}$, we draw the part corresponding to $G$ with straight lines as above. To extend it to the whole arrangement we have to add the arcs crossing the line at infinity that were omitted. These arcs connect pairs of boundary vertices of $P$; to each pseudoline of the arrangement there corresponds a pair of boundary vertices. It cannot happen that some line has only one common vertex with $P$, since in that case all the pseudolines would share a common vertex (this is not possible for monotone quasiline arrangements without digons). We connect these pairs of boundary vertices with straight lines and omit the parts of them in the interior of $P$, to obtain the missing arcs. In that way we assure that there are no bends at infinity. Note that any pair of pseudolines can have at most one common vertex of $P$, since there are no digons in $\mathcal{A}$. Therefore all the lines are distinct. The order in which the pseudolines enter $P$ is the same as the order in which they leave $P$ and this order is therefore reflected in the order of the straight lines that represent them. That also means that no two of these straight lines are parallel and they intersect only in the interior (these parts are omitted) or in the vertices of $P$ since $P$ is convex. The polygonal quasiline arrangement that we obtained therefore has no bends. Since the orders of lines around each crossing is preserved, it is homeomorphic to the original quasiline arrangement by [14, Theorem 3.3.1].

\section{Quasi-topological incidence structures}

In this section the class of quasi-topological configurations based on quasiline arrangements is introduced in a way parallel to topological configurations that are based on pseudoline arrangements. An incidence structure is quasi-topological if its points are points in the projective plane, and lines are pseudolines that form a quasiline arrangement. A quasi-topological incidence structure is monotone if its underlying quasiline arrangement is monotone. A quasi-topological incidence structure $\mathcal{Q}$ is a quasi-topological realization of a combinatorial incidence structure $\mathcal{C}$ if the underlying combinatorial incidence structure of $\mathcal{Q}$ is isomorphic to $\mathcal{C}$.

The notions of combinatorial and topological equivalence for quasi-topological incidence structures are the same as for topological incidence structures. However, since the 
pseudolines are allowed to cross more than once, we extend the notion of mutation equivalence. We will also consider as admissible mutations the local transformations where one pseudoline moves across another pseudoline such that they form a digon and no other pseudolines are crossed.

Figure 7 (a) shows a monotone quasi-topological realization of the $\left(7_{3}\right)$ combinatorial configuration that cannot be realized as a topological configuration; it is polygonal with three bends. However, the quasi-topological realization of the $\left(7_{3}\right)$ configuration from Figure 7 (b) with a 7-fold rotational symmetry is not a monotone quasi-topological configuration, since in every monotone orientation of the pseudolines, for some of the pseudolines the crossings will be in the opposite order.

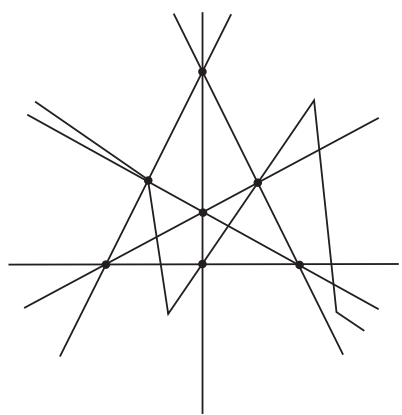

(a)

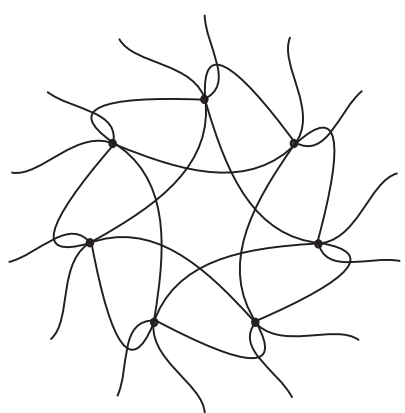

(b)

Figure 7: Two different quasi-topological realizations of the $\left(7_{3}\right)$ configuration.

By Theorem 4.1 all monotone quasi-topological incidence structures can be represented with wiring diagrams (where only a subset of the crossings of the wiring diagram are considered as the points of the incidence structure). Obviously, a topological incidence structure is also monotone quasi-topological. The following theorem therefore shows that the class of monotone quasi-topological incidence structures is in a sense the least generalization of the class of topological incidence structures.

Theorem 8.1. A quasi-topological incidence structure can be represented by a generalized wiring diagram if and only if it is monotone.

Proof. Let $\mathcal{Q}$ be a quasi-topological incidence structure. Suppose it can be represented by a generalized wiring diagram, i.e., it is topologically equivalent to the monotone quasiline arrangement that corresponds to the generalized wiring diagram. Then it must be monotone itself.

Conversely, let $\mathcal{Q}$ be monotone. Then the underlying quasiline arrangement $\mathcal{A}$ is monotone and it can be represented by a generalized wiring diagram by Theorem 4.1.

The following result is a consequence of Theorem 7.3.

Theorem 8.2. Let $\mathcal{Q}$ be a monotone quasi-topological incidence structure. Then $\mathcal{Q}$ is topologically equivalent to a monotone polygonal quasi-topological incidence structure with no bends if and only if the underlying quasiline arrangement contains no digons. 


\section{Combinatorial incidence structures as quasiline arrangements}

Not every lineal combinatorial incidence structure can be realized as a topological incidence structure. Such examples are the well-known configurations $\left(7_{3}\right)$, the Fano plane, and $\left(8_{3}\right)$, the Möbius-Kantor configuration. In this section we show that every combinatorial incidence structure can be realized as a monotone quasi-topological incidence structure. In view of Theorem 8.1, monotone quasi-topological incidence structures are in a sense the least generalization of topological incidence structures with the property that any combinatorial incidence structure has a realization within this class.

Theorem 9.1. Every combinatorial incidence structure can be realized as a monotone quasi-topological incidence structure in the projective plane. Moreover, the order of pseudolines that come to each point of the quasi-topological incidence structure may be prescribed.

Proof. Let $\mathcal{C}$ be a combinatorial incidence structure and let $v$ be the number of points and $n$ be the number of lines of $\mathcal{C}$. Without loss of generality we may number the lines of $\mathcal{C}$ by numbers $1, \ldots, n$. To each point of $\mathcal{C}$ we assign a substring $M_{i}$ of $\{1, \ldots, n\}$, which corresponds to the incident lines of that point in the prescribed order; if the order of lines around points is not prescribed, it may be arbitrary. We find a quasi-topological realization of $\mathcal{C}$ in the following way:

- Let $\pi_{1}, \ldots, \pi_{v}$ be permutations of $\{1, \ldots, n\}$ with the property that elements from $M_{i}$ appear consecutively in $\pi_{i}$. Let $\pi_{i}^{\prime}$ be obtained from $\pi_{i}$ by reversing the order of elements from $M_{i}$. Let $\pi_{0}^{\prime}$ be the identity permutation and $\pi_{v+1}$ the reverse permutation on $\{1, \ldots, n\}$.

- Form the sequence $\pi_{0}^{\prime}, \pi_{1}, \pi_{1}^{\prime}, \ldots, \pi_{v}, \pi_{v}^{\prime}, \pi_{v+1}$; if $\pi_{i}^{\prime}=\pi_{i+1}$, take just one of them. If $\pi_{i}^{\prime} \neq \pi_{i+1}$, insert a sequence of permutations between them to obtain a generalized allowable sequence, for $i=0, \ldots v$. This can always be done, in a bubble sort like manner by interchanging two adjacent numbers in every step.

- A generalized allowable sequence corresponds to a generalized wiring diagram which in turn corresponds to a monotone quasi-topological incidence structure. The points of the incidence structure correspond to the crossings $M_{i}, i=1, \ldots, v$.

The proof of Theorem 9.1 in fact provides us with an algorithm to construct an actual quasi-topological representation of a given combinatorial incidence structure.

We now consider the number of crossings in a quasiline arrangement. Given a quasiline arrangement $\mathcal{A}$, let $p$ be a crossing of $\mathcal{A}$. We define the local crossing number of $p$ to be $\left(\begin{array}{l}k \\ 2\end{array}\right)$ if $k$ lines cross at $p$. Observe that the crossing number of a regular crossing is 1 . The crossing number of the quasiline arrangement $\mathcal{A}$ is the sum over all crossing numbers of its vertices. Given a quasi-topological incidence structure, every pair of pseudolines has at least one point in common. If such a point is not a point of the incidence structure, we call it an unwanted crossing.

Proposition 9.2. Given a topological $\left(n_{k}\right)$ configuration, where all the unwanted crossings are regular, the number of unwanted crossings is $\left(\begin{array}{l}n \\ 2\end{array}\right)-n\left(\begin{array}{l}k \\ 2\end{array}\right)$. 
The number of unwanted crossings in a quasi-topological incidence structure, obtained as in the proof of Theorem 9.1, can be high. However, if the incidence structure has $v$ points and $n$ lines, the number of crossings is at most $(v+1) n^{2}$. Observe that the total number of crossings also gives an upper bound for the maximal number of crossings between two pseudolines. The following two problems are therefore natural to consider.

Problem 9.3. For a given combinatorial incidence structure determine the minimal number of crossings to realize it as a monotone quasi-topological incidence structure in the projective plane.

Problem 9.4. For a given combinatorial incidence structure determine the smallest number $c$ such that it is realizable as a monotone quasi-topological incidence structure in the projective plane with the maximal number of crossings between every pair of pseudolines smaller than $c$.

A quasi-topological configuration obtained from the proof of Theorem 9.1 can have many digons. Is it possible to avoid digons? Is it any easier if we only consider lineal incidence structures?

Problem 9.5. Is every lineal combinatorial incidence structure realizable as a polygonal quasi-topological incidence structure in the projective plane with no bends?

Problem 9.6. Which combinatorial incidence structures are realizable as polygonal quasitopological incidence structures in the projective plane with no bends?

If all the unwanted crossings are straight and there are no bends, a polygonal quasitopological incidence structure is determined by the coordinates of the original vertices (and the directions at which the pseudolines approach infinity). Therefore also the following two problems are of interest.

Problem 9.7. Is every lineal combinatorial incidence structure realizable as a polygonal quasi-topological incidence structure in the projective plane with no bends and all the unwanted crossings straight?

Problem 9.8. Is every topological incidence structure topologically equivalent to a polygonal topological incidence structure in the projective plane with no bends and all the unwanted crossings straight?

\section{Quasi-topological incidence structures as curves on surfaces}

A curve arrangement is a collection of simple closed curves on a given surface such that every pair of curves has at most one point in common at which they cross transversely. In addition the arrangement should be cellular, i.e., the complement of the curves is a union of open discs; see J. Bokowski and T. Pisanski [3].

In this section we show that to any quasi-topological incidence structure we can associate a map $M$ on a closed surface $S$ that can be used to distinguish between mutation classes of quasi-topological configurations. Such a map defines an arrangement of curves on the surface $S$; i.e., every quasi-topological incidence structure can be viewed as a curve arrangement on some surface. This is a generalization of the work of J. Bokowski and R. Strausz [4], where only topological configurations were considered. For the background 
on graphs and maps we refer the reader to B. Mohar and C. Thomassen [14] or J. L. Gross and T. W. Tucker [10].

Let $\mathcal{Q}$ be a quasi-topological incidence structure. We define a graph $G=(V, E)$ corresponding to $\mathcal{Q}$ in the following way. The vertices of $V$ are the points of $\mathcal{Q}$. Two vertices are connected by an edge if the corresponding points of $\mathcal{Q}$ are consecutive on some pseudoline (the intersection points of pseudolines that are not points of the incidence structure are ignored). Note that $G$ is Eulerian since every pseudoline contributes two edges through a vertex. For each vertex $v$ we choose a cyclic order $\pi_{v}$ of edges around $v$. The cyclic orders of all the vertices form a rotation system $\pi=\left\{\pi_{v} ; v \in V\right\}$. Now define a signature mapping $\lambda: E \rightarrow\{1,-1\}$ in the following way. For each edge $e=u v$ we check if the local orientations at $u$ and $v$, determined by the cyclic order of edges around them, agree if we move from $u$ to $v$ along $e$. If they agree we set $\lambda(e)=1$, otherwise $\lambda(e)=-1$. The pair $\Pi=(\pi, \lambda)$ is an embedding scheme of $G$. This defines a map, we denote it by $M=M(\mathcal{Q})$, on some surface $S$. This map is uniquely determined, up to homeomorphism; see B. Mohar and C. Thomassen [14, Theorem 3.3.1]. Note that surface $S$ is non-orientable. This can be seen as follows. Take a cycle $c$ of the map $M$ that corresponds to a pseudoline. Since this is an orientation-reversing curve, starting at a vertex $v$ and traveling along $c$, after returning to $v$ the orientation at $v$ is reversed. That means that there must be an odd number of edges on $c$ with negative signature. Consequently the embedding of $M$ is non-orientable by [14, Lemma 4.1.4].

A straight-ahead walk or a SAW in an Eulerian map is a walk that always passes from an edge to the opposite edge in the rotation at the same vertex; see T. Pisanski et al. [16]. Since the pseudolines of $\mathcal{Q}$ cross transversally at each crossing of $\mathcal{Q}$, every pseudoline corresponds to a straight-ahead walk in the map $M(\mathcal{Q})$. We have shown the following.

Theorem 10.1. For any quasi-topological incidence structure $\mathcal{Q}$ with the set of points $\mathcal{P}$ and the set of lines $\mathcal{L}$ there exists an Eulerian map $M=M(Q)$ on a closed surface, with skeleton $G=(V, E)$ such that $\mathcal{P}=V$, and each $S A W$ is a simple closed curve that corresponds to a line from $\mathcal{L}$.

The maps corresponding to quasi-topological incidence structures can be used to distinguish between quasi-topological incidence structures that are not mutation equivalent.

Theorem 10.2. If two quasi-topological incidence structures $\mathcal{Q}_{1}$ and $\mathcal{Q}_{2}$ are mutation equivalent, then $M\left(\mathcal{Q}_{1}\right)=M\left(\mathcal{Q}_{2}\right)$.

Proof. Admissible mutations do not change the cyclic order of the pseudolines around any crossing that is a point of the incidence structure. On the other hand, the cyclic orders of the pseudolines around every vertex defines the rotation systems for maps $M\left(\mathcal{Q}_{1}\right)$ and $M\left(\mathcal{Q}_{2}\right)$. Moreover, if we choose local rotations consistently, also the embedding schemes are equal. Two maps with equal embedding schemes are considered to be the same.

Example 10.3. Consider the quasi-topological configurations from Figure 7. The corresponding maps have 7 vertices and 21 edges. The map corresponding to the quasitopological configuration on the left has six faces of length three and three faces of length 8 , so it has Euler characteristic -5 and thus it has nonorientable genus equal to 7 . The map corresponding to the quasi-topological configuration on the right has seven faces of length five and one face of lenght seven, so it has Euler characteristic - 6 and thus it has nonorientable genus equal to 8 . Therefore the two quasi-topological configurations are not mutation equivalent. 
For a given quasi-topological incidence structure $\mathcal{Q}$ the map $M(Q)$ can be viewed as a curve arrangement on some nonorientable surface. Since every combinatorial incidence structure can be realized as a quasi-topological incidence structure, we can define the (non)orientable genus of the incidence structure $\mathcal{C}$ as the smallest $g=g(\mathcal{C})$ for which there exists a (non)orientable surface of genus $g$ on which $\mathcal{C}$ can be represented with a curve arrangement.

Problem 10.4. For a given combinatorial incidence structure determine its (non) orientable genus.

\section{Acknowledgements}

The authors would like to thank Sašo Strle for the fruitful discussions regarding the topological aspects of the paper. This work was supported in part by 'Agencija za raziskovalno dejavnost Republike Slovenije', Grants P1-0294, L1-4292, N1-0011 and N1-0032.

\section{References}

[1] J. Bokowski, B. Grünbaum and L. Schewe, Topological configurations $\left(n_{4}\right)$ exist for all $n \geq$ 17, European J. Combin. 30 (2009), 1778-1785, doi:10.1016/j.ejc.2008.12.008, http:// dx.doi.org/10.1016/j.ejc.2008.12.008.

[2] J. Bokowski and V. Pilaud, Enumerating topological $\left(n_{k}\right)$-configurations, Comput. Geom. 47 (2014), 175-186, doi:10.1016/j.comgeo.2012.10.002, http://dx.doi.org/10.1016/ j.comgeo.2012.10.002.

[3] J. Bokowski and T. Pisanski, Oriented matroids and complete-graph embeddings on surfaces, $J$. Combin. Theory Ser. A 114 (2007), 1-19, doi:10.1016/j.jcta.2006.06.012, http: / / dx . doi . org/10.1016/j.jcta.2006.06.012.

[4] J. Bokowski and R. Strausz, A manifold associated to a topological $\left(n_{k}\right)$ configuration, Ars Math. Contemp. 7 (2014), 479-485.

[5] J. Bokowski and B. Sturmfels, Computational synthetic geometry, volume 1355 of Lecture Notes in Mathematics, Springer-Verlag, Berlin, 1989, doi:10.1007/BFb0089253, http:// dx.doi.org/10.1007/BFb0089253.

[6] N. Chiba, K. Onoguchi and T. Nishizeki, Drawing plane graphs nicely, Acta Inform. 22 (1985), 187-201, doi:10.1007/BF00264230, http://dx.doi.org/10.1007/BF00264230.

[7] S. Felsner and H. Weil, Sweeps, arrangements and signotopes, Discrete Appl. Math. 109 (2001), 67-94, doi:10.1016/S0166-218X(00)00232-8, http://dx.doi.org/10 . 1016/ S0166-218X(00)00232-8.

[8] J. E. Goodman, Pseudoline arrangements, in: J. E. Goodman and J. O'Rourke (eds.), Handbook of discrete and computational geometry, CRC, Boca Raton, FL, CRC Press LLC, Boca Raton, FL, pp. 97-128, 2004.

[9] J. E. Goodman and R. Pollack, Semispaces of configurations, cell complexes of arrangements, J. Combin. Theory Ser. A 37 (1984), 257-293, doi:10.1016/0097-3165(84)90050-5, http: //dx.doi.org/10.1016/0097-3165(84)90050-5.

[10] J. L. Gross and T. W. Tucker, Topological graph theory, John Wiley \& Sons, Inc., New York, 1987.

[11] B. Grünbaum, Arrangements and spreads, American Mathematical Society, Providence, R.I., 1972. 
[12] B. Grünbaum, Configurations of points and lines, volume 103 of Graduate Studies in Mathematics, American Mathematical Society, Providence, RI, 2009, doi:10.1090/gsm/103, http: //dx.doi.org/10.1090/gsm/103.

[13] F. W. Levi, Geometrische Konfigurationen, Hirzel, Leipzig, 1929.

[14] B. Mohar and C. Thomassen, Graphs on surfaces, Johns Hopkins University Press, Baltimore, MD, 2001.

[15] T. Pisanski and B. Servatius, Configurations from a graphical viewpoint, Birkhäuser Advanced Texts, Birkhäuser, New York, 2013, doi:10.1007/978-0-8176-8364-1, http://dx.doi . org/10.1007/978-0-8176-8364-1.

[16] T. Pisanski, T. W. Tucker and A. Žitnik, Straight-ahead walks in Eulerian graphs, Discrete Math. 281 (2004), 237-246, doi:10.1016/j.disc.2003.09.011, http://dx. doi.org/10 . $1016 / j . d i s c .2003 .09 .011$.

[17] H. Schröter, Über die bildungsweise und geometrische konstruction der konfigurationen $\left(10_{3}\right)$, Nachr. Ges. Wiss. Göttingen (1889), 193-236.

[18] E. Steinitz, Über die Konstruktion der Konfigurationen $n_{3}$, Ph.D. thesis, Breslau, 1894. 